\title{
Postoperative local incision analgesia for acute pain treatment in patients with hepatocellular carcinoma
}

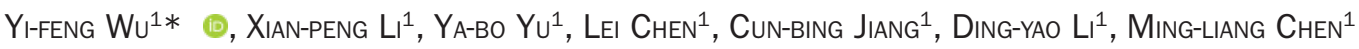

Study conducted at the Department of General Surgery, Yinzhou Hospital Affiliated to Medical School of Ningbo University, Ningbo, Zhejiang, China

Article received: $4 / 26 / 2017$ Accepted for publication: 6/26/2017 *Correspondence: Department of General Surgery, Yinzhou Hospital Affiliated to Medical School of Ningbo University Ningbo, Zhejiang - China Postal code: 315040 yivenw@163.com

http://dx.doi.org/10.1590/1806-9282.64.02.175

\section{SUMmARY}

Objective: The present study aimed to investigate the analgesic effect and safety of using local incision analgesia to treat acute postoperative pain in patients with hepatocellular carcinoma (HCC).

Method: A cohort of 60 patients undergoing liver cancer resection was randomly divided into three groups ( $\mathrm{n}=20$ per group): local incision analgesia (LIA) group, which received local infiltration with ropivacaine combined with a postoperative analgesia pump; intravenous patient-controlled analgesia (PCA) group, which received fentanyl intravenous analgesia postoperatively; and the control group, which received tramadol hydrochloride injection postoperatively according to the NRS scoring system. The postoperative analgesic effect in each group was compared and tumor recurrence (survival) was analyzed using the Kaplan-Meier method.

Results: NRS scores, rate of analgesic usage, ambulation time (h) and intestinal function recovery time (h) were significantly reduced in LIA group compared with the control group at each postoperative time point $(6,12,24$ and 48 hours; $\mathrm{p}<0.05)$. Additionally, the NRS scores of LIA patients at 12 hours post-surgery was significantly reduced compared with PCA group $(\mathrm{p}<0.05)$, and the occurrence of postoperative adverse events in LIA group was significantly lower than that in PCA group $(\mathrm{p}<0.05)$. Survival analysis demonstrated that the mean survival time (tumor recurrence) was significantly increased in LIA group compared with the control group $\left(\chi^{2}=4.749 ; \mathrm{p}=0.029\right)$.

Conclusion: Local incision analgesia improves the analgesic effect, causes fewer adverse reactions and increases postoperative survival time. Our study demonstrated that local incision analgesia is a safe and effective method of postoperative pain management following hepatectomy.

Keywords: Analgesia. Carcinoma, Hepatocellular. Pain, Postoperative. Survival Analysis.

\section{INTRODUCTION}

Hepatocellular carcinoma (HCC) is an extremely malignant tumor that exhibits poor prognosis worldwide. Surgical intervention remains the most effective treatment for HCC, ${ }^{1}$ however, open surgery to remove HCC tumors yields large surgical wounds, long incisions and excessive tissue damage, which can lead to severe acute postoperative pain. The pathophysiological changes induced by acute postoperative pain can cause systemic stress responses and immune function disorders; impact the circulatory, respiratory, digestive and endocrine systems; and increase postoperative com- plications. All of these are unfavorable events with poor prognosis., ${ }^{2,3}$ Therefore, improving the management of postoperative acute pain in patients with liver cancer is important for postoperative rehabilitation. Previous studies suggest that ${ }^{4-6}$ acute postoperative pain is predominantly caused by the surgical incision and that incision pain control is important for postoperative analgesia. The present study investigated the analgesic effect and safety of local incision analgesia in the treatment of acute postoperative pain in patients with HCC, providing clinical insight into the application of local incision analgesia. 


\section{Method}

\section{Setting}

We conducted a blinded, parallel-group randomized controlled trial comparing the analgesic effect and safety of the three different methods of postoperative analgesia in patients undergoing hepatectomy in the Yinzhou Hospital Affiliated to the Medical School of Ningbo University. This work was approved by the ethics committee of the hospital and all patients signed an informed consent form.

\section{Patients}

Patients ( $\mathrm{n}=60)$ who underwent scheduled hepatectomy between January 2014 and January 2016 were selected as subjects for the current study. All subjects were recruited through mass media advertisements, contact with professional groups, presentations at public events and a practice-based database, undergoing laparotomy with incision below the right costal margin. Exclusion criteria were: tumor status III or higher according to the American Society of Anesthesiologists; < 18 years of age; contraindication for ropivacaine or any other local anesthetics; presence of chronic pain; regular consumption of nonsteroidal anti-inflammatory drugs or opioids; psychiatric history; and cardiopulmonary dysfunction.

\section{Randomization and blinding}

The patients were randomized and divided into three groups according to the methods of postoperative analgesia: local incision analgesia group (LIA group; $n=20$ ), intravenous patient-controlled analgesia group (PCA group; $\mathrm{n}=20$ ) and the control group (control group; $\mathrm{n}=20$ ). For randomization, opaque and sealed envelopes were numbered consecutively, and a physician who was not involved in the trial kept the randomization list in a locked drawer until the trial was over and all follow-ups had been conducted. All patients were masked to the treatment groups assigned for the study.

\section{Intervention}

A total of 60 patients underwent tumor resection of the right liver. Of these, 20 patients had liver tumor located in the V and VI hepatic segments, 20 patients had their liver tumor located in the VI and VII hepatic segments, and 20 patients had their liver tumor located on the $V$ and VII hepatic segments. In all of the patients, the incision was made below the right costal margin, with a wound measuring approximately $25 \mathrm{~cm}$.

For treatment of the LIA group, $50 \mathrm{~mL} 0.25 \%$ ropivacaine was applied to the surrounding area and skin, the muscular layer and musculoaponeurotic layer of the inci- sion using abdominal wall infiltration blocks. Simultaneously, a porous catheter was inserted through the skin at the incision site and connected to an external elastic transfusion pump (model TJPS120-1-100-2) filled with $250 \mathrm{~mL}$ $0.25 \%$ ropivacaine. The solution was released continuously at a $5 \mathrm{~mL} / \mathrm{h}$ flow rate through the porous catheter. In the intravenous PCA group, patients were administered fentanyl solution (1.2-1.5 mg fentanyl and $10 \mathrm{mg}$ tropisetron, diluted to $100 \mathrm{~mL}$ using $0.9 \%$ sodium chloride) using an intravenous pump device set with a $2 \mathrm{~mL} / \mathrm{h}$ flow rate and 15 min locking time with $0.5 \mathrm{~mL}$ self-controlled solution. PCA patients received analgesia for two days. The control group (control group) received tramadol hydrochloride injections as postoperative analgesia following the numerical rating scale (NRS) scoring system.

The occurrence of other adverse reactions, including skin pruritus, respiratory depression, sleepiness and hepatic dysfunction were also observed and recorded. Patients themselves determined the pruritus of skin by their feelings, while doctors surveyed the incision exudation and sleepiness. ALT, a glutamic-pyruvic transaminase, is cited as the most sensitive monitoring indicator of liver function damage by the World Health Organization (WHO). Thus, ALT value was used to determine hepatic dysfunction.

All operations were performed using the same technique by the same surgeon with the patient under general anesthesia. Patient follow-up was conducted from the first day of surgical treatment until January 2016 in order to record time of survival (recurrence).

\section{Outcomes}

The primary outcome was rate of analgesia of all the patients at 6, 12, 24 and 48 hours postoperatively, which was recorded according to NRS scoring system ${ }^{7}$ (0-10 points). The scoring system used was as follows: 0 , painless; $1-3$, mild pain, does not affect sleep; 4-6, moderate pain, affects sleep but is tolerable; 7-10, severe pain, intolerable and with lack of sleep.

Secondary outcomes included the adverse effects of analgesia and indicators of rehabilitation. Postoperative nausea and vomiting were recorded using the following scale: 1 , no nausea or vomiting; 2 , nausea, no vomiting; 3 , nausea and vomiting. Adverse reactions, such as pruritus, respiratory depression, sleepiness or hepatic dysfunction were also observed. Indicators of rehabilitation, including the start time of off-bed activity, recovery time of bowel function and status of incision healing were recorded.

\section{Statistical analysis}

Statistical analysis of the data was performed using SPSS 17.0 software (SPSS, Inc., Chicago, IL, USA). Data are 
presented as the mean \pm standard deviation or a percentage. Comparisons between two groups were performed using an independent sample t-test. Enumeration data are described as rate and were analyzed using Pearson's Chi-square test. $\mathrm{p}<0.05$ was considered to indicate a statistically significant difference. The Kaplan-Meier method was used to conduct survival analysis.

\section{Results}

Sixty (60) patients were recruited in the current study, and there was no patient lost at follow-up until hospital discharge (Figure 1). Among the three patient groups, no significant differences were found with respect to the gender, age, average height, average weight, average length of incision or average operating time ( $p>0.05$; Table 1$)$. Postoperative NRS scores at 6, 12, 24 and 48 hours in both LIA group and
PCA group were significantly lower than those in the control

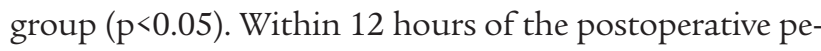
riod, the NRS score in LIA group was significantly lower than that in PCA group $(\mathrm{p}<0.05)$; however, there were no significant differences between the NRS scores of LIA group and PCA group ( $>0.05$; Table 2$)$ at the later time points (24 and 48 hours), which indicates that the effect of local incision analgesia was better than intravenous patient-controlled analgesia within 12 hours postoperatively. The nausea and vomiting scores of LIA group and the control group were $1.40 \pm 0.59$ and $1.50 \pm 0.68$, respectively, which were significantly lower than PCA group $(2.20 \pm 0.77)(p<0.05)$; however, there was no significant difference between the scores of LIA group and the control group ( $p>0.05$; Table 2).

The use of additional intramuscular injections of pain relievers, the start time of off-bed activity and the recovery

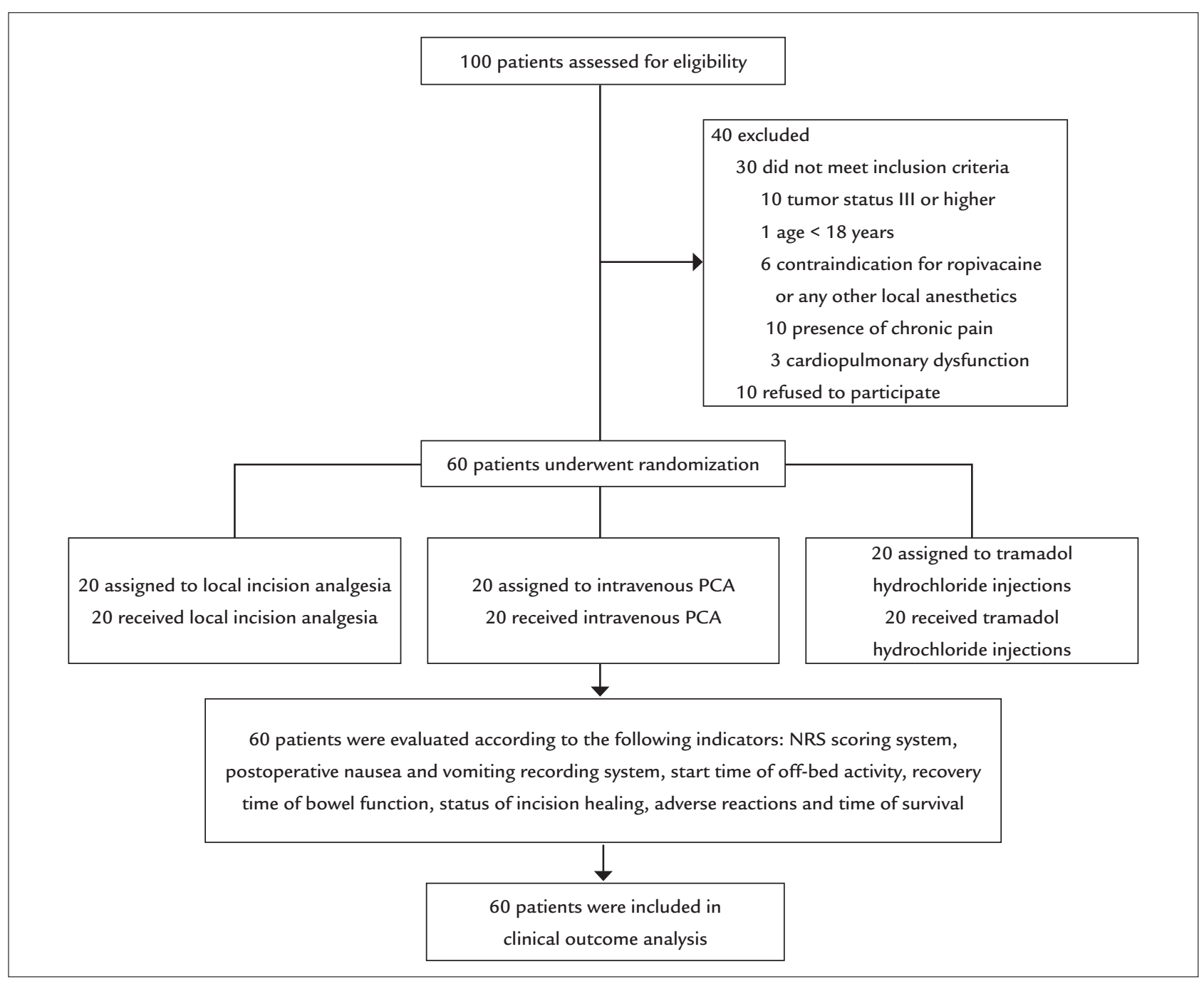

FIGURE 1 Flowchart of patient enrollment, allocation, follow-up and analysis. PCA: patient-controlled analgesia. 
TABLE 1 General characteristics of patients*.

\begin{tabular}{lllllll} 
Group & Male/female $(\mathbf{n})$ & Age (year) & Height $(\mathbf{c m})$ & Weight $(\mathbf{k g})$ & Length of incision $(\mathbf{c m})$ & Operative time $(\mathbf{m i n})$ \\
\hline LIA & $15 / 5$ & $47.61 \pm 10.26$ & $165.89 \pm 6.53$ & $63.58 \pm 9.71$ & $20.35 \pm 1.31$ & $150.10 \pm 24.31$ \\
\hline PCA & $14 / 6$ & $46.92 \pm 10.60$ & $163.72 \pm 6.82$ & $65.21 \pm 8.71$ & $20.01 \pm 1.52$ & $152.21 \pm 25.11$ \\
\hline Control & $16 / 4$ & $47.14 \pm 10.38$ & $164.27 \pm 5.97$ & $64.38 \pm 9.23$ & $20.24 \pm 1.20$ & $149.19 \pm 26.51$ \\
\hline
\end{tabular}

*Data are presented as mean $\pm S D(X \pm S D)$.

LIA: local incision analgesia; PCA: patient-controlled analgesia.

TABLE 2 Scores of pain (NRS system) and nausea and vomit of the three groups $(X \pm S)$.

\begin{tabular}{lllllll} 
Group & $\mathbf{n}$ & Postoperative $\mathbf{6} \mathbf{h}$ & Postoperative 12 $\mathbf{h}$ & Postoperative 24 $\mathbf{h}$ & Postoperative $48 \mathbf{h}$ & Nausea and vomit score \\
\hline A & 20 & $1.95 \pm 0.99^{\mathrm{a}, \mathrm{b}}$ & $1.70 \pm 0.66^{\mathrm{a}, \mathrm{b}}$ & $1.55 \pm 0.51^{\mathrm{a}}$ & $1.25 \pm 0.44^{\mathrm{a}}$ & $1.40 \pm 0.59^{\mathrm{b}}$ \\
\hline B & 20 & $3.15 \pm 10.9^{\mathrm{a}}$ & $2.90 \pm 0.97^{\mathrm{a}}$ & $1.75 \pm 0.55^{\mathrm{a}}$ & $1.45 \pm 0.51^{\mathrm{a}}$ & $2.20 \pm 0.77$ \\
\hline C & 20 & $6.35 \pm 0.99$ & $6.20 \pm 0.95$ & $5.35 \pm 0.88$ & $3.95 \pm 0.69$ & $1.50 \pm 0.68^{\mathrm{b}}$ \\
\hline
\end{tabular}

Compared with group $C,{ }^{\mathrm{a}} \mathrm{p}<0.05$; compared with group $\mathrm{B},{ }^{\mathrm{b}} \mathrm{p}<0.05$.

time of bowel function in LIA group and PCA group were significantly different from that of the control group $(p<0.05)$, while there was no significant difference between the two treatment groups ( $p>0.05$ ). In LIA patients, four cases of incision exudation were observed, which was a significant increase compared with PCA group and the control group $(\mathrm{p}<0.05)$. However, timely detection of incision exudation ensured that no wound infection occurred and all wounds healed to grade A. The occurrence of other adverse reactions, including skin pruritus, respiratory depression, sleepiness and hepatic dysfunction (ALT values) were significantly higher in PCA patients compared with LIA and control groups $(\mathrm{p}<0.05)$, whereas no significant differences were detected between LIA group and the control group ( $p>0.05$; Table 3 ).

Long-term follow-up was conducted for all 60 patients with HCC after surgery. Survival analysis demonstrated that the mean survival time (tumor recurrence) was $20.50 \pm 2.62$ months for LIA and $20.40 \pm 3.64$ months for PCA, with no statistically significant difference between the two groups $\left(\chi^{2}=0.012 ; \mathrm{p}=.911\right)$. However, the mean survival time of patients in the control group was $11.22 \pm 0.86$ months, which was significantly reduced compared with LIA $\left(\chi^{2}=4.749 ; \mathrm{p}=0.029\right)$ and PCA $\left(\chi^{2}=3.904\right.$; $\mathrm{p}=0.048$ (Figure 2).

\section{Discussion}

In addition to temperature, pulse, respiration and blood pressure, the control of postoperative acute pain has attracted increasing attention. In fact, control of postoperative pain remains a challenging problem. Postoperative acute pain is an acute noxious stimulation, predominantly resulting from direct damage of the nerve endings at surgical incision sites and inflammation caused by tissue damage. Acute pain can cause complications including physical trauma and psychological stress, induce coronary heart disease, lung infections, digestive dysfunction and deep vein thrombosis. Postoperative pain can also cause negative emotions, such as anxiety, fear, and depression, leading to disorders of the nervous, circulatory and immune systems, thereby delaying recovery and increasing medical costs. ${ }^{8}$ Appropriate postoperative analgesia may prevent or reduce the occurrence of acute pain and associated conditions, improving postoperative recovery and quality of life. Therefore, the selection of analgesic methods is drawing increasing attention from surgeons. Currently, the most commonly used method of analgesia is an intravenous PCA pump, which is usually infused with opioid narcotic drugs, such as fentanyl. PCA pumps provide good analgesic effects that alleviate postoperative acute pain; however, they may cause adverse reactions, including respiratory depression, nausea and vomiting, sleepiness, cutaneous pruritus and hepatic dysfunction. ${ }^{9,10}$ The results of the present study demonstrated that, compared with a control group, NRS scores in PCA patients gradually decreased over time, confirming the evident analgesic effects of PCA pumps. However, the incidence of postoperative adverse reactions, including nausea and vomiting, cutaneous pruritus, respiratory depression, sleepiness and hepatic dysfunction were significantly higher in PCA patients compared with the control group, indicating the potential risks of using a PCA pump as postoperative analgesic. These findings corroborate the results of studies previously reported by other authors. ${ }^{10,11}$

In our investigation, the efficacy and safety of incision local ropivacaine analgesia for patients undergoing hepa- 
TABLE 3 Postoperative clinical indicators.

\begin{tabular}{|c|c|c|c|c|c|c|c|c|c|}
\hline Group & $\mathbf{n}$ & $\begin{array}{l}\text { Incision } \\
\text { exudation } \\
(n, \%)\end{array}$ & $\begin{array}{l}\text { Respiratory } \\
\text { depression } \\
(n, \%)\end{array}$ & $\begin{array}{l}\text { Sleepiness } \\
(n, \%)\end{array}$ & $\begin{array}{l}\text { Skin } \\
\text { pruritus } \\
(n, \%)\end{array}$ & $\begin{array}{l}\text { Intramuscular } \\
\text { injection } \\
\text { tramadol (n, \%) }\end{array}$ & $\begin{array}{l}\text { Off-bed } \\
\text { activity } \\
\text { time (h) }\end{array}$ & $\begin{array}{l}\text { Bowel function } \\
\text { recovery time }(h)\end{array}$ & $\begin{array}{l}\text { ALT value } \\
(\mathrm{U} / \mathrm{L})\end{array}$ \\
\hline LIA & 20 & $4(20)^{a, b}$ & $0(0)^{\mathrm{b}}$ & $2(10)^{b}$ & $1(5)^{\mathrm{b}}$ & $3(15)^{\mathrm{a}}$ & $40.24 \pm 3.23^{\mathrm{a}}$ & $70.54 \pm 7.72^{\mathrm{a}}$ & $393.45 \pm 128.99^{b}$ \\
\hline PCA & 20 & $0(0)$ & $7(35)$ & $8(40)$ & $6(30)$ & $4(20)^{a}$ & $41.88 \pm 3.04^{\mathrm{a}}$ & $71.71 \pm 5.97^{\mathrm{a}}$ & $490.00 \pm 143.99$ \\
\hline Control & 20 & $0(0)$ & $1(5)^{b}$ & $1(5)^{b}$ & $0(0)^{b}$ & $19(95)$ & $63.91 \pm 7.49$ & $100.64 \pm 10.07$ & $396.90 \pm 126.47^{b}$ \\
\hline
\end{tabular}

Compared with control group, ${ }^{\mathrm{a}} \mathrm{p}<0.05$; compared with PCA group, ${ }^{\mathrm{b}} \mathrm{p}<0.05$.

LIA: local incision analgesia; PCA: patient-controlled analgesia; ALT: alanine aminotransferase.

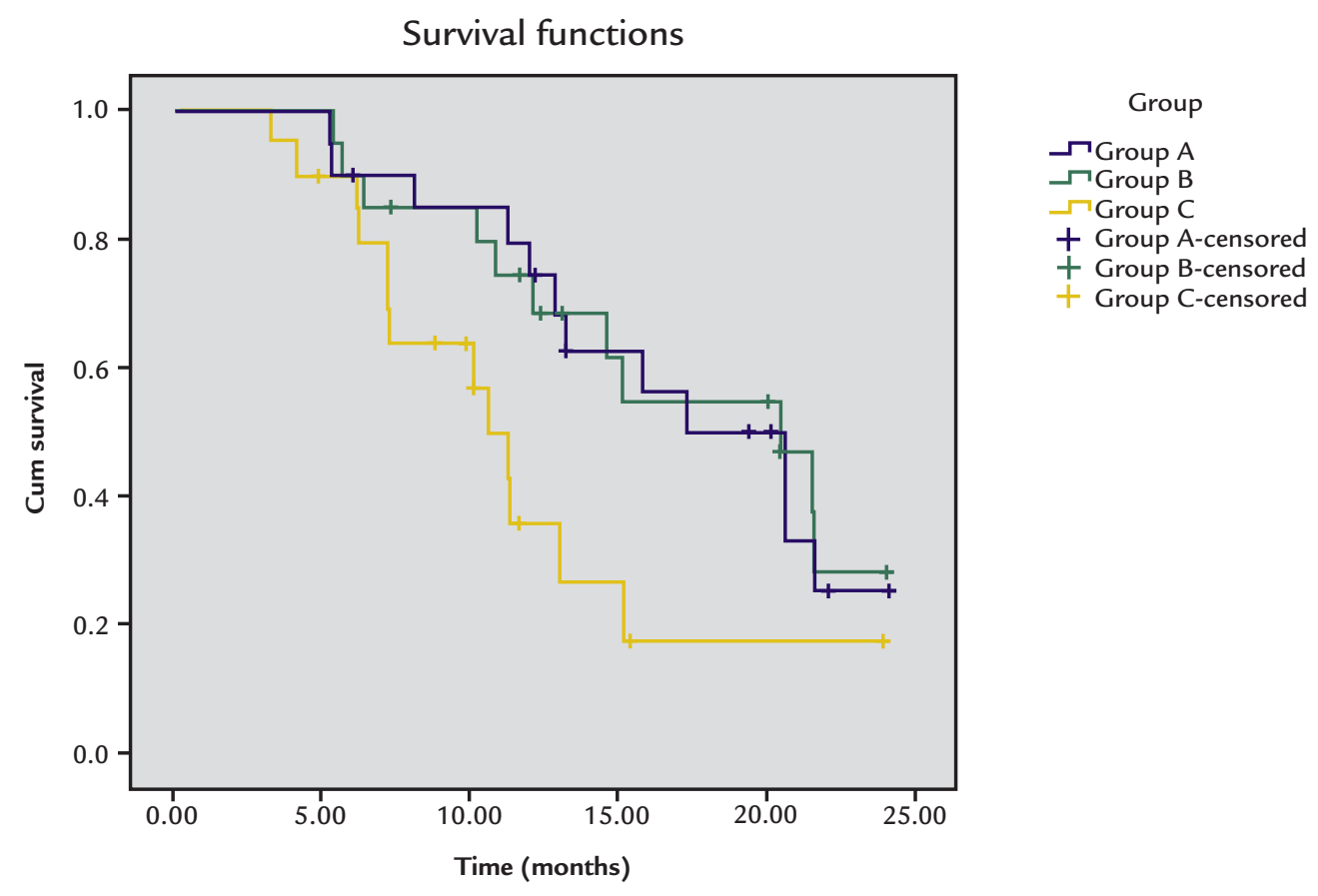

FIGURE 2 Postoperative survival curves (tumor recurrence time) of various treatment groups.

tectomy was evaluated by comparing the postoperative analgesic effect and adverse reactions following open hepatectomy. Ropivacaine is a long-acting local amide anesthetic with low neurotoxicity and cardiotoxicity. Ropivacaine is effective in alleviating postoperative incision pain, improving patient satisfaction with pain management and reducing the incidence of postoperative nausea and vomiting. ${ }^{12-14}$ The present study using local infiltration with ropivacaine combined with a postoperative analgesia pump demonstrated a significantly increased analgesic effect in LIA patients compared with the control group. Compared with PCA group, the analgesic effect in LIA group was significantly higher at 12 hours after surgery. However, by 24 and 48 hours the analgesic effects in LIA group and B were not significantly different. Postoperative adverse reac- tions, including nausea and vomiting, cutaneous pruritus, respiratory depression, sleepiness and hepatic dysfunction were significantly reduced in LIA patients compared with the PCA group, suggesting that analgesia using ropivacaine may block the outer peripheral pain receptors and directly act on the peritoneum, muscle and fascia. Furthermore, this method may reach the areas with increased innervation, such as the subcutaneous tissues, and thus exert a more direct, rapid and efficient analgesic effect on postoperative acute pain. Additionally, ropivacaine reduced the side effects and overall impact on the patients. By reducing the impact of pain on breathing and circulation, this method enables the effective control of the incision pain during the early recovery of the patients, contributing to timely ambulation and early recovery of gastrointestinal function. 
By promoting the timely rehabilitation of patients, it is also in line with the concept of fast-track surgery. ${ }^{15}$

Hepatic dysfunction is one of the most common postoperative complications following hepatectomy. The present study demonstrated that ALT levels of patients in PCA group were significantly higher than the scores in LIA group and control group at 24 hours $(\mathrm{p}<0.05)$. There was also a significant difference between the ALT levels of LIA group and the control group. This suggests that the systemic administration of opioids may interfere with the antioxidant defense system of the liver, triggering apoptosis in hepatocytes and elevation of liver enzymes. ${ }^{16}$ As such, patients with liver cancer are at increased risk of hepatic dysfunction. Since intravenous PCA using opioids such as fentanyl increases the risk of hepatic damage leading to hepatic dysfunction or even liver failure, surgery should simultaneously remove HCC and the normal liver segment. Therefore, the local analgesic method using local incision infiltration combined with an incision analgesia pump may reduce the postoperative hepatic dysfunction and the incidence of complications. It should be noted, however, that postoperative hepatic dysfunction in patients with liver cancer is caused by the combined effect of various factors, including size of liver tumor that is removed, size of the remaining healthy liver tissue, duration of intraoperative hepatic portal occlusion, extent of invasion of the tumor and other perioperative indicators. Thus, to fully realize the benefits of incision local ropivacaine analgesia, the interactions and mechanisms of the various factors that causing postoperative hepatic dysfunction should be further explored.

Our study also demonstrated that the incidence of incision exudation in LIA group was significantly higher than the incidence in groups B and C. However, no incision infections were observed, as the incisions of patients in all LIA group achieved grade A healing. This indicates that the incision local analgesic method provides a safe and effective postoperative analgesic effect without affecting the healing of the surgical incision. The increase in the incidence of incision exudation in LIA group may be due to inadequate drainage, as postoperative incision infections are largely caused by local accumulation of inflammatory substances and tissue debris. The exudates were diverted out of the incision site via the porous penetration catheter of the analgesia pump, and wound dressings were frequently replaced to keep the incision clean and reduce the risk of infections. Kaplan-Meier analysis demonstrated that the use of local incision analgesia prolonged the postoperative survival time until tumor recurrence compared with the control group. This effect may be due to reduced im- mune dysfunction caused by improved control of the postoperative acute pain. Further research is, however, required to understand the mechanisms of such effects.

\section{Conclusion}

Incision local analgesia using ropivacaine provides an improved analgesic effect and increases the postoperative survival time of patients with HCC. Furthermore, the use of incision local analgesia results in fewer adverse reactions and complications, and increases patient satisfaction. Thus, incision local analgesia is a safe and effective analgesic method for managing postoperative acute pain in patients with HCC.

\section{References}

1. Jia WD. Key techniques in precise hepatectomy for hepatocellular carcinoma. Chin J General Surg. 2014; 23(1):1-5.

2. Phelps P, Cakmakkaya OS, Apfel CC, Radke OC. A simple clinical maneuver to reduce laparoscopy-induced shoulder pain: a randomized controlled trial. Obstet Gynecol. 2008; 111(5):1155-60.

3. Estes JD, Reilly C, Trubey CM, Fletcher CV, Cory TJ, Piatak M Jr, et al Antifibrotic therapy in simian immunodeficiency virus infection preserves CD4+ T-cell populations and improves immune reconstitution with antiretroviral therapy. J Infect Dis. 2015; 211(5):744-54.

4. Meechan JG. Pain control in local analgesia. Eur Arch Paediatr Dent. 2009; 10(2):71-6

5. Tighe PJ, Nickerson P, Fillingim RB, Rashidi P. Characterizations of temporal postoperative pain signatures with symbolic aggregate approximations. Clin J Pain. 2017; 33(1):1-11.

6. Huang X, Deng R, Tu W, Hu Z. Dexmedetomidine reduces neuropathic pain in a rat model of skin/muscle incision and retraction. Asian J Surg. 2017; 40(1):35-40.

7. Brunelli C, Zecca E, Martini C, Campa T, Fagnoni E, Bagnasco M, et al Comparison of numerical and verbal rating scales to measure pain exacerbations in patients with chronic cancer pain. Health Qual Life Outcomes. 2010; 8:42.

8. Reddi D, Curran N. Chronic pain after surgery: pathophysiology, risk factors and prevention. Postgrad Med J. 2014; 90(1062):222-7.

9. Zhou B, Wang J, Yan Z, Shi P, Kan Z. Liver cancer: effects, safety, and costeffectiveness of controlled-release oxycodone for pain control after TACE. Radiology. 2012; 262(3):1014-21.

10. Binning AR, Przesmycki K, Sowinski P, Morrison LM, Smith TW, Marcus P, et al. A randomised controlled trial on the efficacy and side-effect profile (nausea/vomiting/sedation) of morphine-6-glucuronide versus morphine for post-operative pain relief after major abdominal surgery. Eur J Pain. 2011; 15(4):402-8.

11. Erdogan Kayhan G, Gul M, Kayhan B, Gedik E, Ozgul U, Kurtoglu EL, et al. Dexmedetomidine ameliorates TNBS-induced colitis by inducing immunomodulator effect. J Surg Res. 2013; 183(2):733-41.

12. Chalam KS, Patnaik SS, Sunil C, Bansal T. Comparative study of ultrasoundguided paravertebral block with ropivacaine versus bupivacaine for postoperative pain relief in children undergoing thoracotomy for patent ductus arteriosus ligation surgery. Indian J Anaesth. 2015; 59(8):493-8.

13. Ohgoshi Y, Yokozuka M, Terajima K. [Serratus-intercostal plane block for breast surgery]. Masui. 2015; 64(6):610-4.

14. Greze J, Vighetti A, Incagnoli P, Quesada JL, Albaladejo P, Palombi O, et al. Does continuous wound infiltration enhance baseline intravenous multimodal analgesia after posterior spinal fusion surgery? A randomized, double-blinded, placebo-controlled study. Eur Spine J. 2017; 26(3):832-9.

15. Ansari D, Gianotti L, Schröder J, Andersson R. Fast-track surgery: procedurespecific aspects and future direction. Langenbecks Arch Surg. 2013; 398(1):29-37.

16. Payabvash S, Beheshtian A, Salmasi AH, Kiumehr S, Ghahremani MH, Tavangar SM, et al. Chronic morphine treatment induces oxidant and apoptotic damage in the mice liver. Life Sci. 2006; 79(10):972-80. 